Pacific Journal of Mathematics

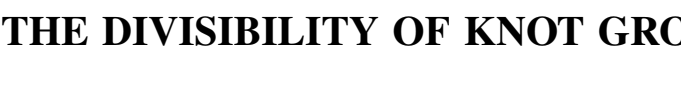




\section{ON THE DIVISIBILITY OF KNOT GROUPS}

\section{Kunio MuRasugi}

Some conditions for the knot group to be an $R$-group, i.e., the group in which the extraction of roots is unique, will be discussed in this paper. In particular, the group of a product knot is an $R$-group iff the knot group of each component is an $R$-group. For a fibred knot, a sufficient condition for its group to be an $R$-group will be given.

A group $G$ is called an $R$-group if for every pair of elements $x$ and $y$, and every natural number $n$, it follows from $x^{n}=y^{n}$ that $x=y$. In other words, $G$ is an $R$-group if $G$ has not more than one solution for every equation $x^{n}=a$. If $G$ is an $R$-group, $G$ is locally infinite. The converse, however, need not be true even if $G$ is restricted to the group of a knot in $S^{3}$. For example, let $G$ be the group of $K(m, n)$, the torus knot of type $(m, n) . \quad G$ has a presentation $G=\left(a, b: a^{m}=b^{n}\right)$. Then the equation $x^{m}=a^{m}$ has infinitely many distinct solutions, $x=a,(b a) a(b a)^{-1},(b a)^{2} a(b a)^{-2}, \cdots$.

This observation gives immediately a negative answer to Problem $\mathrm{N}$ in [3]. Neuwirth asks if a knot group can be ordered. In fact, the group of $K(m, n)(|m|,|n| \geqq 2)$ cannot be ordered, since an ordered group is always an $R$-group. Therefore, Problem N now leads slightly weaker problems: Can a knot group other than torus knot groups be ordered? Or, is a knot group other than torus knot groups an $R$-group?

The purpose of this paper is to give a sufficient condition for the group of a fibred knot to be an $R$-group. (See Theorem 2.) Using this condition, we can prove, for example, that the group of the figure eight knot is an $R$-group. (See Proposition 3 or Proposition 5.)

1. Statement of main results. To make our statement precise, we will introduce some concepts relevant to an $R$-group.

Definition 1. Let $n>1$ be an integer. A group $G$ is said to be $n$-divisible if for any pair of elements $x$ and $y$ in $G$ it follows from $x^{n}=y^{n}$ that $x=y$.

Therefore, a group $G$ is an $R$-group if $G$ is $n$-divisible for every $n$. However, $n$ may be restricted to a prime number. In fact, we have the following easy

Proposition 1. $G$ is mn-divisible iff $G$ is $m$-and $n$-divisible. 
Proposition 1 implies

Proposition 2. A group $G$ is an $R$-group iff $G$ is p-divisible for every prime $p$.

The group of $K(m, n)$ is neither $m$-divisible nor $n$-divisible. However, it will be shown in $\S 4$ that it is $p$-divisible iff $(n, p)=$ $(m, p)=1$, (see Theorem 3).

Now the following theorem shows that we need only consider the groups of prime knots.

THEOREM 1. Let $K$ be the product knot of two knots $K_{1}$ and $K_{2}$. Let $G, G_{1}$, and $G_{2}$ denote the groups of knots $K, K_{1}$, and $K_{2}$, respectively. Then $G$ is an $R$-group iff $G_{1}$ and $G_{2}$ are $R$-groups.

The proof will be given in $\S 2$.

Finally, the main theorem of this paper is stated as follows.

THEOREM 2. Let $K$ be a nontrivial fibred (or Neuwirth) knot. Suppose that the Alexander polynomial $\Delta(t)$ of $K$ has no repeated roots and let $\alpha_{1}, \cdots, \alpha_{m}$ be all the roots of $\Delta(t)$. If the group of $K$ is not p-divisible, $p$ a prime, then the multiplicative subgroup generated by $\alpha_{1}, \cdots, \alpha_{m}$ in the complex number field contains a nontrivial pth root of unity.

The proof will be given in $\S 3$.

2. Proof of Theorem 1. Since a subgroup of an $R$-group is an $R$-group, "only if" part of Theorem 1 is trivial.

To prove "if" part, we will show a slightly stronger theorem below.

Let $G=G_{1} \underset{H}{*} G_{2}$ denote the free product of two groups $G_{1}$ and $G_{2}$ with an amalgamated subgroup $H$.

Let $\mathbb{S}_{i}(i=1,2)$ be the system of right coset representatives for $G_{i}$ modulo $H$. Then any element $x$ of $G$ has a unique normal form: $x=h x_{1} \cdots x_{k}$, where $h \in H$, each $x_{i}(\notin H)$ belongs to only one system $\mathbb{S}_{1}$ or $\mathbb{S}_{2}$ and no two successive elements $x_{i}$ and $x_{i+1}(i=1, \cdots, k-1)$ belong to the same system. $k$ is the length of $x$, denoted by $l(x)$. $h$ will be called the initial factor of $x$, and is denoted by $i(x)$. If $x \in H$, or if $l(x) \geqq 1$ and if $x_{k}$ and $x_{1}$ belong to different systems, $x$ is said to be cyclically reduced.

THEOREM 1A. $G=G_{1}^{*} G_{2}$ is an $R$-group if the following condition (1)-(3) are satisfied: 
(2.1) (1) $G_{1}$ and $G_{2}$ are $R$-groups,

(2) $H$ is an isolated subgroup of $G_{1}$ and $G_{2}$. In other words, if $l(x) \leqq 1$ and $l\left(x^{n}\right)=0$ for some natural number $n$, then $l(x)=0$.

(3) $x^{n}=y^{n}$ for some natural number $n$ yields $i(x)=i(y)$.

Theorem 1A implies immediately

CoRollary. $G=G_{1} * G_{2}$ is an $R$-group iff $G_{1}$ and $G_{2}$ are groups.

The proof of Theorem 1A will follow from Lemmas 1-6 below.

Lemma 1. Let $y$ be a noncyclically reduced element of $G=G_{1 H} G_{2}$ and $y \notin H$. Then $y$ can be written as.

$$
y=u^{-1} y_{0} u,
$$

where

(2.2) (1) $y_{0}$ is a cyclically reduced element of $G$. Let $y_{0}=h z_{1} \cdots z_{k}$, $h \in H$, be the normal form of $y_{0}$. Then $l\left(y_{0}\right)=k \geqq 1$.

(2) $u$ has the normal form, $u=\widetilde{h} u_{1} \cdots u_{\lambda}$, where $\widetilde{h} \in H, \lambda=l(u) \geqq 1$, and $u_{i} \in \mathbb{B}_{1}$ or $\mathbb{B}_{2}$.

(3) (i) If $k \geqq 2$, then $k$ is even, and $z_{k}$ and $u_{1}$ are not in the same system, and $u_{1}^{-1} \widetilde{h}^{-1} h z_{1} \notin H$. Therefore, $l(y)=k+2 \lambda-1$.

(ii) If $k=1$, then $z_{1}$ and $u_{1}$ are not in the same system and $l(y)=2 \lambda+1$.

Since the proof is straightforward, it will be omitted, or see [2, $\S 4.2]$. Denote $k=\widetilde{l}(y)$.

In the following lemmas, we assume that the group $G=G_{1 H}^{*} G_{2}$ satisfies (2.1) (1)-(3).

LEMMA 2. Let $y$ be a noncyclically reduced element of $G=G_{1 H}^{*} G_{2}$ and $y \notin H$. Then for any positive integer $n, l\left(y^{n}\right)$ is given as follows: (2.3) (1) If $\widetilde{l}(y)=k \geqq 2$ and $l(u)=\lambda(\geqq 1)$, then $l\left(y^{n}\right)=n k+2 \lambda-$ $1 \geqq 2$.

(2) If $\tilde{l}(y)=1$, then $l\left(y^{n}\right)=2 \lambda+1 \geqq 3$.

Proof. (1) is obvious.

To prove (2), let $y=u^{-1} y_{0} u$ be the form obtained in Lemma 1 . Since $\widetilde{l}(y)=1$, we see that $y_{0}=h z, z \in G_{1}-H$ or $G_{2}-H$. Since $z$ and $u_{1}$ are not in the same factor, it follows that

$$
l\left(y^{n}\right)=l\left(u^{-1}(h z)^{n} u\right) \leqq 2 \lambda+1 .
$$

An inequality in (2.4) holds only when $(h z)^{n}$ and $u_{1}$ belong to the 
same factor, i.e., $(h z)^{n} \in H$. Then by (2.1) (2) $h z \in H$, and hence, $z \in H$. This is a contradiction. Therefore, $l\left(y^{n}\right)=2 \lambda+1$.

LEMMA 3. Let $x$ and $y$ be cyclically reduced elements of $G$. If $x^{n}=y^{n}$, then $l(x)=l(y)$.

Proof. First we note that for any cyclically reduced element $z$

$$
l\left(z^{n}\right) \leqq 1 \quad \text { iff } \quad l(z) \leqq 1 .
$$

Now the proof will be divided into the following three cases.

Case 1. $l(x) \geqq 2$.

Then $\quad l\left(x^{n}\right)=n l(x) \geqq 2 . \quad$ Since $\quad x^{n}=y^{n}, l\left(y^{n}\right)=l\left(x^{n}\right)=n l(x) \geqq 2$ and hence $l(y) \geqq 2$. Therefore, $l\left(y^{n}\right)=n l(y)$. Now, the conclusion of Lemma 3 is immediate.

Case 2. $l(x)=1$.

Then $l\left(x^{n}\right)=1$, and hence, $l\left(y^{n}\right)=1$, since $x^{n}=y^{n}$. Therefore, it follows from (2.5) that $l(y) \leqq 1$. $l(y)$ cannot be 0 , otherwise $l\left(y^{n}\right)$ would be 0 . Therefore, $l(y)=1=l(x)$.

Case 3. $l(x)=0$.

Then $l\left(x^{n}\right)=0$ and hence $l\left(y^{n}\right)=0$. Then it follows from (2.1) (2) that $l(y)=0$.

LEMMA 4. Let $x$ and $y$ be cyclically reduced elements of $G$. Then $x^{n}=y^{n}$ yields $x=y$.

Proof. By Lemma 3, we know that $l(x)=l(y)$. Let $x=h x_{1} \cdots x_{k}$ and $y=g y_{1} \cdots y_{k}$ be the normal forms of $x$ and $y$.

Case 1. $l(x)=k \geqq 2$.

The normal forms of $x^{n}$ and $y^{n}$ can be written as

$$
x^{n}=h^{\prime} x_{1}^{(n-1)} x_{2}^{(n-1)} \cdots x_{k}^{(n-1)} \cdots x_{1}^{\prime} x_{2}^{\prime} \cdots x_{k}^{\prime} x_{1} \cdots x_{k}
$$

and

$$
y^{n}=g^{\prime} y_{1}^{(n-1)} y_{2}^{(n-1)} \cdots y_{k}^{(n-1)} \cdots y_{1}^{\prime} y_{2}^{\prime} \cdots y_{k}^{\prime} y_{1} \cdots y_{k} .
$$

Since $x^{n}=y^{n}$, we obtain that $x_{1}=y_{1}, \cdots, x_{k}=y_{k}$. Moreover, it follows from (2.1) (3) that $i(x)=h=i(y)=g$. Therefore, $x=h x_{1} \cdots$ $x_{k}=g y_{1} \cdots y_{k}=y$.

Case 2. $l(x)=k=1$.

Then $x=h x_{1}$ and $y=g y_{1}$. Suppose that $x_{1}$ and $y_{1}$ are not in 
the same system. Then $x^{n}=\left(h x_{1}\right)^{n}=\left(g y_{1}\right)^{n}=y^{n}$ must belong to $H=G_{1} \cap G_{2}$, and hence, $x$ belongs to $H$ by (2.1)(2). It contradicts our assumption. Therefore, $x_{1}$ and $y_{1}$ belong to the same system. Since $G_{1}$ and $G_{2}$ are $R$-groups, $x=y$ follows.

Case 3. $l(x)=l(y)=0$.

Then $x=y$, since $H$ is an $R$-group as a subgroup of $G_{i}$.

Lemma 5. Let $x$ and $y$ be elements of $G$. Suppose that only one of $x$ and $y, x$ say, is cyclically reduced, but $y$ is not, and hence $y \notin H$. Then $x^{n} \neq y^{n}$.

Proof. Case 1. $l(x) \geqq 2$.

It follows from Lemma 2 that $l\left(x^{n}\right) \equiv 0(\bmod 2)$, while $l\left(y^{n}\right) \equiv$ $1(\bmod 2)$. Therefore, $x^{n} \neq y^{n}$.

Case 2. $l(x)=1$.

Since $x$ belongs to one factor, we see that $l\left(x^{n}\right)=1$. On the other hand, $l\left(y^{n}\right) \geqq 2$ by Lemma 2 . Therefore, $x^{n} \neq y^{n}$.

Case 3. $l(x)=0$. Suppose $x^{n}=y^{n}$.

Then $x^{n}$ belongs to $H$ and so does $y^{n}$. Therefore, $y$ belongs to $H$ by (2.1) (2). It contradicts our assumption.

Lemma 6. Suppose that neither $x$ nor $y$ is cyclically reduced. Then $x^{n}=y^{n}$ yields $x=y$.

Proof. By Lemma 1, we can write

$$
x=u^{-1} x_{0} u \text { and } y=v^{-1} y_{0} v,
$$

where $x_{0}$ and $y_{0}$ are cyclically reduced. Then $x^{n}=y^{n}$ yields $u^{-1} x_{0}^{n} u=$ $v^{-1} y_{0}^{n} v$ and hence,

$$
x_{0}^{n}=u v^{-1} y_{0}^{n} v u^{-1}=\left(u v^{-1} y_{0} v u^{-1}\right)^{n} .
$$

Since $x_{0}$ is cyclically reduced, it follows from Lemmas 4 and 5 that $x_{0}=u v^{-1} y_{0} v u^{-1}$. It implies that $x=y$.

Theorem 1A follows immediately from these lemmas.

Now what it remains to show is that the group of the product knot $K_{1} \# K_{2}$ satisfies (2.1)(1)-(3). 
Let $G_{i}=G\left(K_{i}\right)$ be the knot group of $K_{i}$. Then it is well known that $G=G_{1 H}^{*} G_{2}$, where $G=G\left(K_{1} \# K_{2}\right)$ and $H$ is the infinite cyclic group generated by $t$ that is represented by a meridian. Denote $P^{\prime}$ the commutator subgroup of a group $P$.

Now (2.1)(1) is exactly the assumption of Theorem 1.

To prove (2.1) (2), take an element $x$ from $G_{1}$, say. $x$ is written as $x=t^{k} x_{1}$, where $x_{1} \in G_{1}^{\prime}$. Then $x^{n}=t^{k n} x_{1}^{\prime}$ for some $x_{1}^{\prime} \in G_{1}^{\prime}$. Therefore $x^{n}=t^{k n} x_{1}^{\prime}=t^{m}$, for some $m$, yields $k n=m$, and hence, $t^{k n} x_{1}^{\prime}=t^{k n}$. Since $G_{1}$ is an $R$-group, it follows that $\left(t^{k} x_{1}\right)^{n}=\left(t^{k}\right)^{n}$ yields $t^{k} x_{1}=t^{k}$. Therefore, $x_{1}=1$, and hence, $x \in H$.

To prove (2.1) (3), let $x=t^{q} x_{1} \cdots x_{k}$ and $y=t^{r} y_{1} \cdots y_{l}$ be the normal forms of $x$ and $y$. Then $x^{n}=t^{q n} g$ and $y^{n}=t^{r n} h$ for some $g, h \in G^{\prime}$. Thus $x^{n}=y^{n}$ implies that $t^{q n}=t^{r n}$ in $G / G^{\prime}$, and hence, $q=r$. This proves (2.1) (3).

3. Proof of Theorem 2. Let $K$ be a fibred knot in $S^{3}$. Then the knot group $G$ of $K$ is a semi-direct product of $Z$ and $F$, where $Z$ is an infinite cyclic group generated by $t$ and $F$ is a free group freely generated by $r$ elements $x_{1}, \cdots, x_{r}$. The action of $Z$ on $F$ is given by an automorphism $\phi$ of $F$ :

$$
\phi: x_{i} \longrightarrow t x_{i} t^{-1}=W_{i}, \quad i=1, \cdots, r .
$$

Let $\left\{F_{n}\right\}$ be the lower central series of $F$, where $F=F_{1}$ and $F_{n}=\left[F, F_{n-1}\right]$. Then $\phi$ induces an automorphism $\phi_{n}: F_{n} \rightarrow F_{n}$ for each $n$, since $F_{n}$ is a characteristic subgroup of $F$. Using $\phi_{n}$, we can construct a semi-direct product $G_{n}(K)$ of $Z$ and $F_{n}$, where the action of $Z$ on $F_{n}$ is given by $\phi_{n}$.

Now we know that $F_{n} / F_{n+1}$ is a free abelian group of finite rank and $\phi_{n}$ induces an automorphism $\hat{\phi}_{n}$ of $F_{n} / F_{n+1}$. Let $\Delta_{n}(t)$ be the characteristic polynomial of the integer matrix $M_{n}$ associated with $\hat{\phi}_{n}$. We should note here that the Alexander polynomial $\Delta_{K}(t)$ of $K$ is just $\Delta_{1}(t)$ defined above.

First we want to know the characteristic roots of $\hat{\phi}_{n}$.

To do this, we use a technique given in [2, §5.7].

Let $A_{0}(Z, r)$ be the graded associative $Z$-algebra freely generated by $r$ elements $y_{1}, y_{2}, \cdots, y_{r}$. We define the bracket product in $A_{0}(Z, r)$ by $[u, v]=u v-v u$. Then there exists a free Lie algebra $\Lambda_{0}(Z, r)$ freely generated by $\xi_{1}, \cdots, \xi_{r}$ that is imbedded in $A_{0}(Z, r)$ equipped with the bracket product [2, Lemma 5.5]. Let $\Lambda_{n}$ be the submodule consisting of all homogeneous elements of degree $n$ in $\Lambda_{0}(Z, r)$. Then $F_{n} / F_{n+1}$ is isomorphic to $\Lambda_{n}$, as an abelian group, under a natural mapping [2, Theorem 5.12]. Therefore, the automorphism $\hat{\phi}_{n}$ of $F_{n} / F_{n+1}$ induces an automorphism $\tilde{\phi}_{n}$ of $\Lambda_{n}$. 
Let $C$ denote the complex number field. For $u_{1}, \cdots, u_{k} \in C-\{0\}$, we denote by $\left\langle u_{1}, \cdots, u_{k}\right\rangle$ the multiplicative subgroup in $C-\{0\}$ generated by $u_{1}, \cdots, u_{k}$.

LEMmA 7. Suppose that $\Delta_{K}(t)$ has no repeated roots, and let $\alpha_{1}, \cdots, \alpha_{r}$ be all the roots of $\Delta_{K}(t)$. Let $\beta_{1}, \cdots, \beta_{s}$ be all the roots of $\Delta_{n}(t)$. Then

$$
\left\langle\alpha_{1}, \cdots, \alpha_{r}\right\rangle \supset\left\langle\beta_{1}, \cdots, \beta_{s}\right\rangle \text {. }
$$

Therefore, in particular, the splitting field of $\Delta_{n}(t)$ is contained in that of $\Delta_{K}(t)$.

Proof. Since we are concerned with only the characteristic roots, we consider the vector space $V_{n}=F_{n} / F_{n+1} \otimes C$ instead of a free abelian group $F_{n} / F_{n+1}$, and the vector space $\Lambda_{n} \otimes C$ instead of $\Lambda_{n}$.

Since $\Delta_{K}(t)$ has no repeated roots, the characteristic polynomial coincides with the minimal polynomial and further $V_{1}$ is completely reducible. Therefore, we can choose a new basis $\left\{\bar{x}_{1}, \cdots, \bar{x}_{r}\right\}$ for $V_{1}$ so that $\hat{\phi}_{1}\left(\bar{x}_{i}\right)=\alpha_{i} \bar{x}_{i}$. The corresponding new basis of $\Lambda_{1} \otimes C$ will be written as $\bar{\xi}_{1}, \cdots, \bar{\xi}_{r}$ and $\tilde{\phi}_{1}\left(\bar{\xi}_{i}\right)=\alpha_{i} \bar{\xi}_{i}$. Then $\tilde{\phi}_{n}$ maps a basis element $\bar{\xi}_{i, n}$ of $\Lambda_{n} \otimes C$ to an element of the form $\alpha_{1}^{k_{1}} \cdots \alpha_{r}^{k} \bar{\xi}_{i, n}$. Therefore, the matrix associated with $\tilde{\phi}_{n}$ is diagonal and each characteristic root $\beta_{i}$ of $\tilde{\phi}_{n}$, and that of $\hat{\phi}_{n}$, is of the form $\alpha_{1}^{k_{1}} \cdots \alpha_{r}^{k_{r}}$. This proves Lemma 7.

Now we proceed to the proof of Theorem 2.

Suppose that $G$ is not $p$-divisible, $p>1$. Then there exist $x$ and $y$ in $G$ such that $x \neq y$ but $x^{p}=y^{p}$. Since $G$ is a semi-direct product of $Z$ and $F, x$ and $y$ can be written as $x=g t^{k}$ and $y=h t^{k}$ for some integer $k$ and for some elements $g$ and $h$ in $F=F_{1}=G^{\prime} . g \neq h$, since $x \neq y$. Therefore, there is an element $u \neq 1$ in $F_{n}-F_{n+1}$, $n \geqq 1$, such that $h=u g$. Then it follows from $x^{p}=x^{p}$ that

$$
\left(g t^{k}\right)^{p}=\left(u g t^{k}\right)^{p} \text {. }
$$

By induction on $p$, it is easy to show that

$$
\left(u g t^{k}\right)^{p} \equiv u\left(t^{k} u t^{-k}\right)\left(t^{2 k} u t^{-2 k}\right) \cdots\left(t^{(p-1) k} u t^{-(p-1) k}\right)\left(g t^{k}\right)^{p}\left(\bmod F_{n+1}\right) .
$$

Compare (3.1) and (3.2) to obtain

$$
u\left(t^{k} u t^{-k}\right)\left(t^{2 k} u t^{-2 k}\right) \cdots\left(t^{(p-1) k} u t^{-(p-1) k}\right) \equiv 1\left(\bmod F_{n+1}\right) .
$$

Consider the semi-direct product $\widetilde{G}_{n}$ of $Z$ and $F_{n} / F_{n+1}$, where the action of $Z$ on $F_{n} / F_{n+1}$ is given by an induced automorphism $\hat{\phi}_{n}$. 
Introduce a new multiplication $m t \cdot g=t g^{m} t^{-1}$ for $t \in Z$ and $g \in F_{n} / F_{n+1}$ so that $\widetilde{G}_{n}$ becomes an $R$-module, where $R$ is the group ring of the infinite cyclic group $Z$. This $R$-module $\widetilde{G}_{n}$ is finitely presented and its relation matrix is $\left\|M_{n}-t I\right\|$.

Then equation (3.3) is interpreted as a relation (3.4) below, which holds in $\widetilde{G}_{n}$ :

$$
\left(1+t^{k}+t^{2 k}+\cdots+t^{(p-1) k}\right) u=0 .
$$

Since $\left\|M_{n}-t I\right\|$ is a relation matrix of the $R$-module $\widetilde{G}_{n}$, some factor of the characteristic polynomial $\Delta_{n}(t)$ of $M_{n}$ must divide $f(t)=1+t^{k}+\cdots+t^{(p-1) k}=\left(1-t^{p k}\right) /\left(1-t^{k}\right)$. Since $p$ is prime, it follows that the set of roots of $A_{n}(t)$ contains a $m p$ th root of unity, $m \mid k$, and hence, it contains a $p$ th root of unity. Theorem 2 now follows form Lemma 7.

COROLlaRY 1. Under the hypothesis of Theorem 2, if the group of a knot $K$ is not $p$-divisible, then the splitting field of $\Delta_{K}(t)$ contains $a$ pth root of unity.

4. Divisibility of the groups of torus knots.

THEOREM 3. The group $G$ of the torus knot $K(m, n)$ is p-divisible, $p$ a prime, iff $(n, p)=(m, p)=1$. In any case, any two solutions of the equation $x^{p}=y^{p}$ are conjugate.

Proof. Suppose that $G$ is not $p$-divisible. We want to show that $p \mid n$ or $p \mid m$.

Since $\Delta(t)=(1-t)\left(1-t^{m n}\right) /\left(1-t^{m}\right)\left(1-t^{n}\right)$, all the roots $\alpha_{1}, \cdots$, $\alpha_{(m-1)(n-1)}$ of $\Delta(t)$ are distinct and they are $m n$th root of unity. Therefore, $\left\langle\alpha_{1}, \cdots, \alpha_{(m-1)(n-1)}\right\rangle$ contains only $m n$th roots of unity. On the other hand, it follows from Theorem 2 that $\left\langle\alpha_{1}, \cdots, \alpha_{(m-1)(n-1)}\right\rangle$ must contain a $p$ th root of unity. Therefore, $p$ must be a divisor of $m n$, and hence, $p \mid m$ or $p \mid n$. To prove the converse, let $G$ have a presentation $\left(a, b: a^{m}=b^{n}\right)$. If $p \mid m$, then $m=p q$, and $b a^{q} b^{-1} \neq a^{q}$, since $a^{m}$ generates the center of $G$. However, $\left(b a^{q} b^{-1}\right)^{p}=a^{p q}$. Therefore, $G$ is not $p$-divisible.

To prove the last part ${ }^{1}$, first we should note that $G=G_{1 H}^{*} G_{2}$, where $G_{1}, G_{2}$, and $H$ are infinite cyclic groups generated by $a, b$, and $c=a^{m}\left(=b^{n}\right)$, respectively, and $H$ is the center of $G$, but $H$ is not an isolated subgroup of $G_{i}, i=1,2$.

Now, suppose $x^{p}=y^{p}$. We may assume without loss of generality that $p \mid m$ and hence, $p \nmid n$, since $(m, n)=1$.

1 I owe the proof mostly to D. Solitar. 
Let $x=h x_{1} \cdots x_{k}$ and $y=g y_{1} \cdots y_{l}$ be the normal forms of $x$ and $y$, where $h=c^{r}=a^{m r}$ and $g=c^{s}=a^{m s}$. By Lemma 6 , one of $x$ and $y, x$ say, is assumed to be cyclically reduced. Further, if $k=1$, we may assume that $x$ is in $G_{1}$.

Case 1. $y$ is cyclically reduced.

It is obvious that $k \geqq 2$ iff $l \geqq 2$ and if $k, l \geqq 2$, then $k=l$. Therefore, $x=y$ follows immediately from $x^{p}=y^{p}$.

Suppose that $k \leqq 1$ and $l \leqq 1$. There are six cases to be considered.
(i) $\left\{\begin{array}{l}x=c^{r} a^{d} \\ y=c^{s} a^{e}\end{array}\right.$
(ii ) $\left\{\begin{array}{l}x=c^{r} a^{d} \\ y=c^{s} b^{f}\end{array}\right.$
(iii) $\left\{\begin{array}{l}x=c^{r} a^{d} \\ y=c^{s}\end{array}\right.$
(iv) $\left\{\begin{array}{l}x=c^{r} \\ y=c^{s} a^{e}\end{array}\right.$
(v) $\left\{\begin{array}{l}x=c^{r} \\ y=c^{s} b^{f}\end{array}\right.$
(vi) $\left\{\begin{array}{l}x=c^{r} \\ y=c^{s}\end{array}\right.$

where $0<d, e<m$, and $0<f<n$.

In Cases (i) and (vi), it is easy to see that $x=y$ follows from $x^{p}=y^{p}$. Case (ii) does not occur. In fact, $x^{p}=y^{p}$ implies that $f p \equiv 0(\bmod n)$ and hence, $f \equiv 0(\bmod n)$, since $(p, n)=1$. Similarly, Case (v) does not occur. Case (iii) also does not occur. In fact, $x^{p}=y^{p}$ implies that $r m p+d p=s m p$, and hence, $d \equiv 0(\bmod m)$. Similarly, Case (iv) does not occur.

Case 2. $y$ is not cyclically reduced.

Suppose that $l(x)=k \geqq 2$. Then $l\left(x^{p}\right)$ is always even, while $l(y)$ is odd by Lemma 2 . Therefore, $x^{p} \neq y^{p}$, and hence, $l(x) \leqq 1$.

Suppose that $l(x) \leqq 1$. Since $y$ is not cyclically reduced, $\widetilde{l}(y) \neq 0$. If $\tilde{l}(y) \geqq 2$, then $l\left(y^{p}\right) \geqq 2$ by Lemma 2. This contradicts $x^{p}=y^{p}$. Therefore, $\widetilde{l}(y)=1$.

Now we have to consider the following four cases.
(i ) $\left\{\begin{array}{l}x=c^{r} a^{d} \\ y=u^{-1} c^{s} a^{e} u\end{array}\right.$
(ii) $\left\{\begin{array}{l}x=c^{r} a^{d} \\ y=u^{-1} c^{s} b^{f} u\end{array}\right.$
(iii) $\left\{\begin{array}{l}x=c^{r} \\ y=u^{-1} c^{s} a^{e} u\end{array}\right.$
(iv) $\left\{\begin{array}{l}x=c^{r} \\ y=u^{-1} c^{s} b^{f} u,\end{array}\right.$

where $0<d, e<m$, and $0<f<n$.

Now Case (ii) does not occur. In fact, $x^{p}=y^{p}$ implies that $c^{r p} a^{d p}=u^{-1} c^{s p} b^{f p} u$ and hence, $b^{f p} \in H$, otherwise $l\left(y^{p}\right) \geqq 2$. Therefore, 
$f p \equiv 0(\bmod n)$. Since $(p, n)=1$, it follows that $f \equiv 0(\bmod n)$. This contradicts $0<f<n$. By a similar argument, we can prove that Case (iv) does not occur. Case (iii) does not occur. In fact $x^{p}=y^{p}$ implies that $a^{r p m}=u^{-1} a^{s p m+e p} u$ and hence, $r p m=s p m+e p$. It shows that $e \equiv 0(\bmod m)$, which contradicts $0<e<m$.

Now consider Case (i). If $\left(c^{s} a^{e}\right)^{p} \notin H$, then $l\left(y^{p}\right) \geqq 2$, while $l\left(x^{p}\right)=1$. Therefore, $\left(c^{s} a^{e}\right)^{p} \in H$. Then $x^{p}=y^{p}$ implies that $a^{r m p+d p}=u^{-1} a^{s m p+e p} u=$ $a^{s p m+e p}$, since $\alpha^{s p m+e p} \in H$. Therefore, $r m p+d p=s p m+e p$ and hence, $r m+d=s m+e$. This shows that $y=u^{-1} x u$.

This proves Theorem 3 .

\section{Applications.}

Proposition 3. The group $G$ of the figure eight knot $K$ is an $R$-group.

Proof. Since $\Delta_{K}(t)=1-3 t+t^{2}$ has two real roots $\alpha$ and $1 / \alpha$, $|\alpha| \neq 1$, it follows that $\langle\alpha\rangle$ contains only real numbers. Therefore, $G$ is $p$-divisible except possibly for $p=2$. If $\langle\alpha\rangle$ contains $-1, \alpha^{n}=$ -1 for some integer $n$. Therefore $|\alpha|=1$, a contradiction. Since $\langle\alpha\rangle$ has no nontrivial roots of unity, it follows from Theorem 2 that $G$ is an $R$-group.

Note that the figure eight knot is the only fibred knot $K$ with $\Delta_{K}(t)=1-3 t+t^{2}$.

Finally we consider the knots whose Alexander polynomials $\Delta(t)$ are of degree $4 .^{2}$ Such a polynomial has the form:

$$
\Delta(t)=(t-\alpha)\left(t-\frac{1}{\alpha}\right)(t-\beta)\left(t-\frac{1}{\beta}\right) .
$$

Note that $\alpha \neq 1$ and $\beta \neq 1$.

Case 1. Both $\alpha$ and $\beta$ are real.

Case 2. Only one of them, $\alpha$ say, is real.

Case 3. Neither $\alpha$ nor $\beta$ is real.

Case 1. $\langle\alpha, \beta\rangle$ contains no nontrivial $p$ th root of unity, except possibly for -1 .

If both $\alpha$ and $\beta$ are positive, then $\langle\alpha, \beta\rangle$ does not contain -1 , and hence, $G$ is an $R$-group.

Case 2. Since $\beta$ and $1 / \beta$ are roots of a quadratic equation $1+a t+t^{2}=0$ for some real number $a$, we see that $1 / \beta=\bar{\beta}$ and $|\beta|=1$. Suppose that $\xi=\alpha^{m}(1 / \alpha)^{n} \beta^{r} \bar{\beta}^{s}$ is a $p$ th root of unity. Then

\footnotetext{
2 I am grateful to R. A. Smith for his helpful suggestion.
} 
$|\xi|=|\alpha|^{m-n}=1$ yields $m=n$, and hence, $\xi=\beta^{r-s}$. Therefore, $\beta$ is a $p(r-s)$ th root of unity. Thus the splitting field of $\Delta(t)$ must contain a $p(r-s)$ th root of unity.

Case 3. If one of the roots has the absolute value 1, so do all the roots. Kronecker's theorem, then, says that all the roots are $m$ th roots of unity for some $m$.

Suppose that $|\alpha| \neq 1$. Since $\alpha$ and $\beta$ are complex roots, $\bar{\alpha}$ and $\bar{\beta}$ are also the roots of $\Delta(t)$. If $\bar{\alpha}=1 / \alpha$, then $|\alpha|=1$, a contradiction. If $\bar{\alpha}=\beta$, then $|\alpha|=|\beta|$, and hence, $|\alpha|=|\beta|=1$, since $|\alpha \beta|=1$. This contradicts our assumption. Therefore, $\bar{\alpha}=1 / \beta$. Suppose that $\langle\alpha, \beta\rangle$ contains an $n$th root of unity $\xi$. We write $\xi=\alpha^{k} \beta^{l}$. Since $\bar{\alpha}=1 / \beta$, we see that $\xi=\alpha^{k} \bar{\alpha}^{-l}$. Then $1=|\xi|=|\alpha|^{k-l}$ yields $k=l$. Therefore, $\xi=(\alpha / \bar{\alpha})^{k}$ and $\alpha / \bar{\alpha}$ is a knth root of unity. Since $\alpha / \bar{\alpha}$ is contained in the splitting field $\mathscr{F}$ of $\Delta(t)$, $\mathscr{F}$ must contain a $k n$th root of unity.

These observations will be collected in the following

Proposition 4. Let $K$ be a fibred knot. Suppose that $\Delta_{K}(t)$ is of degree less than 5 and has no repeated roots. Then

(5.1) (1) If all the roots of $\Delta_{K}(t)$ are positive real numbers, then $G$, the group of $K$, is an $R$-group.

(2) Suppose that $\Delta_{K}(t)$ has only two complex roots $\beta$ and $\bar{\beta}$. If $G$ is not $p$-divisible, then $\beta$ is a pqth root of unity for some $q$, and therefore, the splitting field of $\Delta_{K}(t)$ contains a pqth root of unity.

(3) Suppose that $\Delta_{K}(t)$ has four complex roots $\alpha, \bar{\alpha}, \beta, \bar{\beta}$, and that $|\alpha| \neq 1$. If $G$ is not $p$-divisible, then $\alpha / \bar{\alpha}$ is a kpth root of unity for some $k$, and therefore, the splitting field of $\Delta_{K}(t)$ contains a kpth root of unity.

This proposition will be used to prove Proposition 5 below.

In 1961, Trotter [4] studied the splitting fields of the Alexander polynomials of certain fibred knots. Combining his results [4, p. 557] with corollary, we obtain that

(5.2) (1) The groups of $4_{1}, 6_{2}, 7_{6}, 8_{12}, 9_{42}, 9_{44}, 9_{45}$, are $p$-divisible for all prime $p$ other than 2 ,

(2) The groups of $3_{1}, 6_{3}, 7_{7}, 8_{20}, 8_{21}, 9_{48}$, are $p$-divisible for all prime other than 2 or 3 , or 5 .

(3) The group of $5_{1}$ is $p$-divisible for all prime $p$ other than 2 
Since the divisibility of the groups of $3_{1}, 4_{1}$, and $5_{1}$ has already been determined, we consider the remaining eleven knots.

Now, each of the Alexander polynomials of $8_{12}$ and $9_{45}$ has four positive real roots. Therefore by (5.1) (1), their groups are $R$-groups.

On the other hand, each of the Alexander polynomials of $6_{2}$, $7_{6}$, and $9_{42}$ has only two complex roots. If their groups are not 2-divisible, the splitting fields of their Alexander polynomial must contain $2 q$ th root of unity by (5.1) (2). According to [4, p. 557] they contain only 2 nd root of unity. Therefore, one of the complex roots must be -1 , which cannot occur.

That the group $G$ of $9_{48}$ is an $R$-group will be proved as follows.

Suppose that $G$ is not $p$-divisible, $p=2$ or 3 . Since the splitting field of the Alexander polynomial of $9_{48}$ contains only $2 \mathrm{nd}$, 3rd, or 6 th root of unity, it follows from (5.1) (2) that one of the complex roots, $\beta$ say, is either 2 nd, 3 rd, or 6 th root of unity. However, the calculation shows that

$$
\beta=\frac{7-\sqrt{13}}{4}+\frac{1}{2 \sqrt{2}} \sqrt{7 \sqrt{13}-23} i,
$$

which is none of 2 nd, 3rd and 6 th root of unity.

Therefore, $G$ is an $R$-group.

Finally, we consider the groups of $9_{44}, 6_{3}$, and $7_{7}$. Each of the Alexander polynomials of these knots has four complex roots, each of which does not lie on the unit circle. Therefore, we can apply (5.1) (3) on these groups.

Let $\mathscr{F}(K)$ denote the splitting field of $\Delta_{K}(t)$. Then according to [4, p. 557], we know that

(5.3) (1) $\mathscr{F}\left(9_{44}\right)$ contains only the 2 nd and 4 th roots of unity.

(2) $\mathscr{F}\left(6_{3}\right)$ and $\mathscr{F}\left(7_{7}\right)$ contain only the 2 nd 3 rd, and 6 th roots of unity.

Now a direct computation shows that

$$
\begin{gathered}
\Delta_{9_{44}}(t) \text { has a root } \alpha_{1}=\frac{1}{2}\left\{\left(2+\sqrt{\frac{\sqrt{17}-1}{2}}\right)+i\left(1+\sqrt{\frac{\sqrt{17}+1}{2}}\right)\right\}, \\
\Delta_{6_{3}}(t) \text { has a root } \alpha_{2}=\frac{1}{4}\{(3-\sqrt{\sqrt{52}-5})+i(3+\sqrt{\sqrt{52}+5})\},
\end{gathered}
$$

and

$\Delta_{7_{7}}(t)$ has a root $\alpha_{3}=\frac{1}{4}\{(5+\sqrt{\sqrt{84}+3})+i(3+\sqrt{\sqrt{84}-3})\}$. 
Then it is easy to verify that $\alpha_{1} / \bar{\alpha}_{1}$ is none of the 2 nd and 4 th root of unity, and each of $\alpha_{2} / \bar{\alpha}_{2}$ and $\alpha_{3} / \bar{\alpha}_{3}$ is none of the 2nd, 3rd, and 6 th root of unity.

Therefore, the groups of these three knots are $R$-groups.

This proves the following

Proposition 5. The groups of knots $4_{1}, 6_{2}, 6_{3}, 7_{6}, 7_{7}, 8_{12}, 9_{42}, 9_{44}, 9_{45}$, $9_{48}$ are $R$-groups.

REMARK. (2.1) (2) is not a necessary condition for $G$ to be an $R$-group.

\section{REFERENCES}

1. A. G. Kurosh, The Theory of Groups, II, New York, Chelsea, 1957.

2. W. Magnus, A. Karrass, and D. Solitar, Combinatorial Group Theory, Intersciences, New York, 1966.

3. L. Neuwirth, Knot Groups, Annals of Mathematical Studies 56, Princeton University Press, 1965.

4. H. F. Trotter, Periodic automorphisms of groups and knots, Duke Math. J., 28 (1961), 553-557.

Received February 22, 1973 and in revised form March 18, 1974. This research was supported in part by the Canada Council Leave Fellowship W710027 and NRC Grant No. A4034.

UNIVERSITY OF TORONTO 



\section{PACIFIC JOURNAL OF MATHEMATICS}

EDITORS

RICHARD ARens (Managing Editor)

University of California

Los Angeles, California 90024

\section{R. A. Beaumont}

University of Washington

Seattle. Washington 98105

\section{J. DugundjI}

Department of Mathematics University of Southern California Los Angeles, California 90007

D. Gilbarg and J. Milgram

Stanford University

Stanford. California 94305

\section{ASSOCIATE EDITORS}
E. F. BECKENBACH
B. H. NEUMANN
F. WOLF
K. Yoshida

\section{SUPPORTING INSTITUTIONS}

\author{
UNIVERSITY OF BRITISH COLUMBIA \\ CALIFORNIA INSTITUTE OF TECHNOLOGY \\ UNIVERSITY OF CALIFORNIA \\ MONTANA STATE UNIVERSITY \\ UNIVERSITY OF NEVADA \\ NEW MEXICO STATE UNIVERSITY \\ OREGON STATE UNIVERSITY \\ UNIVERSITY OF OREGON \\ OSAKA UNIVERSITY
}

\author{
UNIVERSITY OF SOUTHERN CALIFORNIA \\ STANFORD UNIVERSITY \\ UNIVERSITY OF TOKYO \\ UNIVERSITY OF UTAH \\ WASHINGTON STATE UNIVERSITY \\ UNIVERSITY OF WASHINGTON

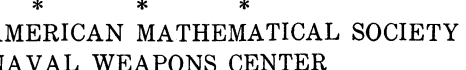

The Supporting Institutions listed above contribute to the cost of publication of this Journal, but they are not owners or publishers and have no responsibility for its content or policies.

Mathematical papers intended for publication in the Pacific Journal of Mathematics should be in typed form or offset-reproduced, (not dittoed), double spaced with large margins. Underline Greek letters in red, German in green, and script in blue. The first paragraph or two must be capable of being used separately as a synopsis of the entire paper. Items of the bibliography should not be cited there unless absolutely necessary, in which case they must be identified by author and Journal, rather than by item number. Manuscripts, in duplicate if possible, may be sent to any one of the four editors. Please classify according to the scheme of Math. Rev. Index to Vol. 39. All other communications to the editors should be addressed to the managing editor, or Elaine Barth, University of California, Los Angeles, California, 90024.

100 reprints are provided free for each article, only if page charges have been substantially paid Additional copies may be obtained at cost in multiples of 50 .

The Pacific of Journal Mathematics is issued monthly as of January 1966. Regular subscription rate: $\$ 72.00$ a year (6 Vols., 12 issues). Special rate: $\$ 36.00$ a year to individual members of supporting institutions.

Subscriptions, orders for back numbers, and changes of address should be sent to Pacific Journal of Mathematics, 103 Highland Boulevard, Berkeley, California, 94708.

\section{PUBLISHED BY PACIFIC JOURNAL OF MATHEMATICS, A NON-PROFIT CORPORATION}

Printed at Kokusai Bunken Insatsusha (International Academic Printing Co., Ltd.), 270, 3-chome Totsuka-cho. Shinjuku-ku, Tokyo 160. Japan.

Copyright (C) 1973 by Pacific Journal of Mathematics Manufactured and first issued in Japan 


\section{Pacific Journal of Mathematics}

\section{Vol. 52, No. $2 \quad$ February, 1974}

Harm Bart, Spectral properties of locally holomorphic vector-valued functions .....

J. Adrian (John) Bondy and Robert Louis Hemminger, Reconstructing infinite

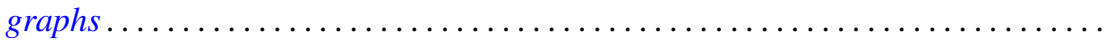

Bryan Edmund Cain and Richard J. Tondra, Biholomorphic approximation of planar

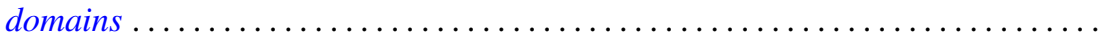

Richard Carey and Joel David Pincus, Eigenvalues of seminormal operators,

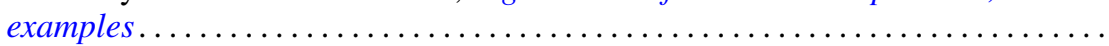

Tyrone Duncan, Absolute continuity for abstract Wiener spaces . . . . . . . . . . . . Joe Wayne Fisher and Louis Halle Rowen, An embedding of semiprime

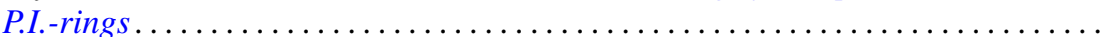

Andrew S. Geue, Precompact and collectively semi-precompact sets of semi-precompact continuous linear operators. . . . . . . . . . . . . . . .

Charles Lemuel Hagopian, Locally homeomorphic $\lambda$ connected plane continua ..... . Darald Joe Hartfiel, A study of convex sets of stochastic matrices induced by

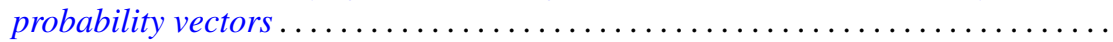

Yasunori Ishibashi, Some remarks on high order derivations $\ldots \ldots \ldots \ldots \ldots \ldots \ldots$ Donald Gordon James, Orthogonal groups of dyadic unimodular quadratic forms.

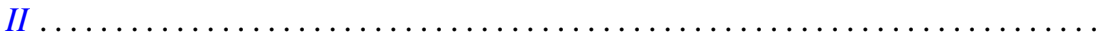

Geoffrey Thomas Jones, Projective pseudo-complemented semilattices . . . . . . . . . Darrell Conley Kent, Kelly Denis McKennon, G. Richardson and M. Schroder,

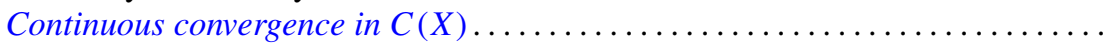

J. J. Koliha, Some convergence theorems in Banach algebras ...

Tsang Hai Kuo, Projections in the spaces of bounded linear oper

George Berry Leeman, Jr., A local estimate for typically real functions . .

475

Andrew Guy Markoe, A characterization of normal analytic spaces by the

homological codimension of the structure sheaf .........

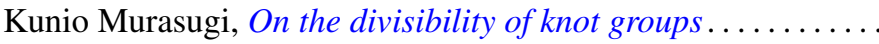

John Phillips, Perturbations of type I von Neumann algebras.

Billy E. Rhoades, Commutants of some quasi-Hausdorff matrices . .

David W. Roeder, Category theory applied to Pontryagin duality

Maxwell Alexander Rosenlicht, The nonminimality of the differential closure .

Peter Michael Rosenthal, On an inversion theorem for the general Mehler-Fock transform pair.

Alan Saleski, Stopping times for Bernoulli automorphisms

John Herman Scheuneman, Fundamental groups of compact complete locally affine complex surfaces. II. ........................

Vashishtha Narayan Singh, Reproducing kernels and operators with a cyclic vector. I. .

Peggy Strait, On the maximum and minimum of partial sums of random variables.

J. L. Brenner, Maximal ideals in the near ring of polynomials modulo 2 .

Ernst Gabor Straus, Remark on the preceding paper: "Ideals in near rings of polynomials over a field" ..........................

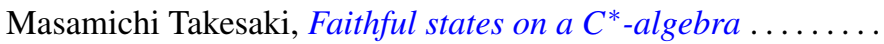

R. Michael Tanner, Some content maximizing properties of the regular simplex.

Andrew Bao-hwa Wang, An analogue of the Paley-Wiener theorem for certain

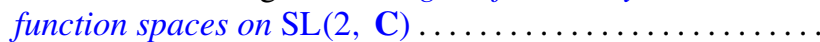

